\title{
Caracterização Elétrica de Filmes Ultrafinos de PANI/PVS: Material Potencial para Detecção de Amônia em Galpões de Criação Avícola
}

\author{
Mirela de C. Santos \\ Departamento de Física, UFV
}

Fabrício A. dos Santos, Felipe P. Teixeira, Gislayne E. Gonçalves, Andrea G. C. Bianchi, Rodrigo F. Bianchi

Departamento de Física, UFOP

\begin{abstract}
Resumo: Nesse trabalho filmes ultrafinos de PANI/PVS foram caracterizados óptica, elétrica e morfologicamente para estudo da viabilidade desses sistemas como elemento ativo de sensores de amônia. Os filmes automontados foram depositados entre microeletrodos metálicos formados por uma fina camada de $\mathrm{NiCr}$ recoberto com Au. Medidas de absorção na região do UV-VIS mostraram o crescimento linear dos filmes, enquanto a análise das imagens de microscopia de força atômica mostrou o aumento da rugosidade, do tamanho de grão e do tamanho da partícula com a espessura dos filmes. A caracterização elétrica dc evidenciou uma significativa mudança na resistência elétrica do sistema quando a espessura do filme tornou-se equivalente à espessura do eletrodo de $\mathrm{NiCr}$. Esse efeito foi atribuído à alta resistência elétrica da interface NiCr-filme. A impedância complexa obtida foi típica de materiais sólidos desordenados, com influência da interface NiCr-filme em baixas frequências. Por fim, os filmes mostraram ainda aumento de resistência elétrica em cerca de três vezes quando expostos às condições ambientais de galpões de criação avícola $\left(\left[\mathrm{NH}_{3}\right]<50 \mathrm{ppm}\right)$, bem como em outras aplicações agropecuárias onde a necessidade do monitoramento desse gás é de grande importância. Esses resultados demonstram o potencial que filmes de PANI/PVS apresentam para aplicações agropecuárias.
\end{abstract}

Palavras-chave: Camada-por-camada, propriedade elétrica, sensor, polímero eletrônico, dispositivo.

\section{Preparation and Characterization of Ultrathin PANI/PVS: Films for Control the Ammonia Level in Poultry Houses}

Abstract: In this work we investigated the optical, electrical and morphological properties of ultrathin PANI/PVS films as active material of ammonia sensor for a poultry house. The layer-by-layer polymer films were deposited onto $\mathrm{NiCr}$ interdigitated microelectrode recovered with $\mathrm{Au}(\mathrm{NiCr} / \mathrm{Au})$ line arrays. The buildup of the multilayers was monitored at each deposition step by UV-VIS spectroscopy while the atomic force microscopy (AFM) was employed to monitor the change in the roughness, in the grain and particle sizes. We observed a significant change in the dc conductivity when the thickness of the films was close to that of the $\mathrm{NiCr}$ layer. This effect is attributed to the higher interfacial electrical resistance between $\mathrm{NiCr}$ and the polymer film, compared to the resistance of the Au-film interface and polymer bulk. The alternating conductivity of the polymer was typical of solid disordered materials, under the influence of the $\mathrm{NiCr}$ electrode at low frequencies. Finally, the electrical sensitivity of the system under the exposure of $\mathrm{NH}_{3}$ reveals PANI/PVS films as a feasible active element for use in poultry house control systems.

Keywords: Layer-by-layer, electrical properties, sensor, electronic polymer, device.

\section{Introdução}

A partir de meados da década de 1970, com a descoberta das propriedades condutivas do trans-poliacetileno ${ }^{[1]}$, polímeros começaram a ser utilizados em diversos dispositivos eletrônicos, passando de materiais passivos (isolantes) para ativos ${ }^{[2]}$. Desde então, inúmeros polímeros condutivos passaram a ser fabricados e seus dispositivos eletrônicos bastante explorados e estudados devido, principalmente, às suas facilidades de preparação e de manufatura aliadas às suas versáteis propriedades ópticas, elétricas e mecânicas, bem como aos seus baixos custos de fabricação e altas durabilidade e eficiências ópticas e/ou elétricas. Entretanto, o curto período entre as descobertas das propriedades semicondutoras e a aplicação desses polímeros em dispositivos eletrônicos deixou controvérsias e questões sobre os efeitos de interface eletrodo-polímero nas propriedades elétricas desses sistemas, evidenciando

Autor para correspondência: Mirela de C. Santos, Departamento de Física, Centro de Ciências Exatas e Tecnológicas, Universidade Federal de Viçosa, Av. Peter Henry Rolfs s/n, Campus Universitário, CEP: 36570-000,Viçosa, MG, Brasil.E-mail: mirela.santos@ufv.br 
a necessidade do estudo criterioso desses efeitos ${ }^{[3-4]}$. Dessa forma, técnicas de caracterização elétrica têm sido utilizadas para a investigação não apenas dos mecanismos de injeção e condução dos portadores de carga ao longo dessas camadas, como também para o estudo dos efeitos de interface e de volume na condutividade elétrica desses materiais, bem como dos efeitos da morfologia ${ }^{[5]}$ dos filmes e dos arranjos geométricos utilizados para a fabricação dos dispositivos poliméricos ${ }^{[6]}$. Nesse contexto, nesse trabalho foram preparados filmes ultrafinos de polianilina/poli(vinil sulfato de sódio) (PANI/PVS) com o objetivo de investigar as propriedades ópticas, elétricas e de morfologia desses sistemas visando, sobretudo, a aplicação potencial desses filmes como sensores para detecção e controle da amônia em galpões de criação avícola ${ }^{[7-8]}$. Da literatura ${ }^{[9]}$ sabe-se que a presença de amônia em concentrações superiores a 5 ppm tem causado impactos desfavoráveis ao bem-estar animal, ao meio ambiente e à saúde dos trabalhadores, além da perda de peso, subdesenvolvimento da carcaça e maior índice de mortalidade das aves. Nesse sentido, o desenvolvimento de dispositivos capazes de detectar e monitorar a amônia nessas condições é um tema atual e de interesse científico e agropecuário.

\section{Experimental}

Filmes ultrafinos de polianilina/poli(vinil sulfato de sódio) (PANI/PVS)foramobtidos sobresubstratodevidropelatécnica de automontagem, ou layer-by-layer ( $L b l)$, que possibilita a fabricação de filmes com espessuras nanométricas (variando de $\sim 3$ a centenas de nanômetros $)^{[10]}$. Filmes nanoestruturados com número de bicamadas $(n)$ de PANI/PVS variando de 1 a 13 foram então depositados sobre substratos de vidro recobertos com microeletrodos interdigitados de $\mathrm{NiCr} / \mathrm{Au}$, para caracterização elétrica, e sobre substratos de vidro, para caracterização óptica e de morfologia de superfície. Detalhes dos microeletrodos interdigitados sobre substrato de vidro são apresentados na Figura 1. Nessa estrutura, a espessura da camada de $\mathrm{NiCr}\left(\mathrm{H}_{\mathrm{NiCr}}\right)$ é de $(20 \pm 3) \mathrm{nm}$, enquanto a de $\mathrm{Au}\left(\mathrm{H}_{\mathrm{Au}}\right)$ é de $(180 \pm 5) \mathrm{nm}$. Já a distância média (L) entre os eletrodos é de $10 \mu \mathrm{m}$ e o comprimento (1) dos microeletrodos de $5 \times 10^{-3} \mathrm{~m}$.

$\mathrm{O}$ crescimento dos filmes foi monitorado a cada deposição por meio de medidas dos seus espectros de absorção na região do ultravioleta-visível. A morfologia e a uniformidade dos filmes

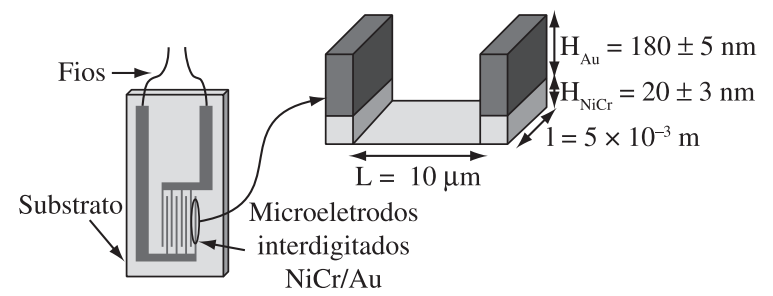

Figura 1. Representação esquemática da estrutura dos microeletrodos interdigitados utilizados para as caracterizações elétricas dos filmes $\mathrm{H}_{\mathrm{Au}}$ e $\mathrm{H}_{\mathrm{NiCr}}$ são, respectivamente, as espessuras das camadas de $\mathrm{NiCr}$ e de $\mathrm{Au}$, enquanto $\mathrm{L}$ e 1 a distância média e o comprimento dos eletrodos. de PANI/PVS foram analisadas por imagens de Microscopia de Força Atômica (Atomic Force Microscopy - AFM) utilizando o Software Digital Nanoscope III. A área de varredura foi de $5 \mu \mathrm{m}$, a uma frequência de $1,2 \mathrm{~Hz}$ com resolução das imagens de 512 pixels, utilizando uma varredura piezo modelo AS-130 (' $\mathrm{J}$ ') por modo de contato intermitente. As curvas de corrente vs. tensão foram obtidas com o eletrômetro Keithley $6517 \mathrm{~A}$ Electrometer/High Resistance Meter, com aplicação de tensão de $0 \mathrm{~V}$ a $1 \mathrm{~V}$, enquanto para as medidas de impedância complexa foi utilizado o Impedanciômetro Solartron 1260 Impedance/Gain Phase Analyser, com aplicação de uma amplitude de tensão de $100 \mathrm{mV}$ em um intervalo de frequência de $1 \mathrm{~Hz}$ a $2 \mathrm{MHz}$. Finalmente, um ambiente com controle de concentração de amônia foi preparado e utilizado para a exposição dos filmes a esse gás mantendo-se, para tanto, as condições usuais de ambientes de criação avícola ${ }^{[11]}$. Nesse sistema, o controle e o monitoramento da concentração de $\mathrm{NH}_{3}$ foram realizados com o detector de amônia, modelo DG200, da empresa Instrutherm.

\section{Resultados e Discussões}

A Figura 2a mostra o gráfico com as curvas de absorção $v s$. comprimento de onda obtido com filmes de PANI/PVS com número de bicamadas ( $n$ ) variando de 1 em 1 até 10 . Observa-se nessa figura que a intensidade da absorção aumenta com $n$ em todo o espectro e seu máximo ocorre em torno de $900 \mathrm{~nm}$. A partir desse resultado foi traçado um gráfico da intensidade da absorção em $900 \mathrm{~nm} v s . n$, Figura $2 \mathrm{~b}$, e observado sua dependência linear, garantindo que a adsorção dos filmes ocorreu como esperado ${ }^{[12]}$.

As imagens de AFM de filmes Lbl de PANI/PVS apresentadas na Figura 3 mostram a superfície da topografia das amostras contendo 1, 4 e 10 bicamadas, depositados sobre substrato de vidro cuja rugosidade média quadrática é da ordem de $3,3 \mathrm{~nm}$. Das imagens obtidas das amostras com $n=1$ e 4 , Figura 3a e b, respectivamente, observa-se que a quantidade de aglomerados aumenta com $n$. Esse aumento pode estar relacionado ao fato que durante as primeiras deposições sobre o substrato de vidro hidrofílico ocorre uma competição entre as moléculas de água e o filme de PANI/PVS no processo de adsorção, conforme discutido por Lobo et $\mathrm{al}^{\left[{ }^{[13]}\right.}$ para um dos derivados da PANI. A Tabela 1 mostra os parâmetros obtidos com o tratamento das imagens com o Software Digital Nanoscope III. Nessa tabela, observa-se que a rugosidade média quadrática, o tamanho médio dos grãos e o tamanho médio das partículas aumentam com o número de

Tabela 1. Parâmetros obtidos a partir das imagens de AFM de filmes com $n=1,4$ e 10 .

\begin{tabular}{cccc}
\hline $\boldsymbol{n}$ & $\begin{array}{c}\text { Tamanho médio } \\
\text { de grãos }\left(\mathbf{n m}^{\mathbf{2}}\right)\end{array}$ & $\begin{array}{c}\text { Tamanho médio } \\
\text { das partículas }(\mathbf{n m})\end{array}$ & $\begin{array}{c}\text { Rugosidade Média } \\
\text { Quadrática }(\mathbf{n m})\end{array}$ \\
\hline 1 & 1948 & 22 & 10 \\
4 & 3796 & 46 & 16 \\
10 & 9381 & 62 & 23 \\
\hline
\end{tabular}


bicamadas, ou seja, com a espessura do filme. Esse resultado pode ser explicado levando-se em consideração dois estágios de crescimento: inicialmente, Figura $3 \mathrm{a}$, as moléculas são depositadas aleatoriamente sobre todo o substrato. Nesse estágio a absorção é favorecida devido às forças de atração entre as moléculas da solução polimérica e o substrato. Esse

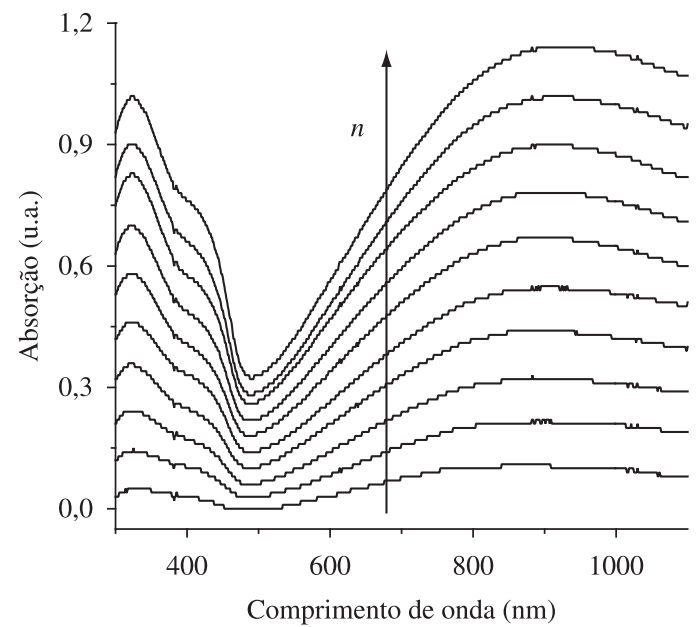

(a) primeiro estágio ocorre durante a deposição das primeiras bicamadas por $L b l$. Em seguida, no segundo estágio, ocorre o rearranjo das moléculas já adsorvidas, levando à formação de grãos maiores e, posteriormente, os sítios não ocupados são propícios ao processo de agregação, como mostra a Figura 3c.

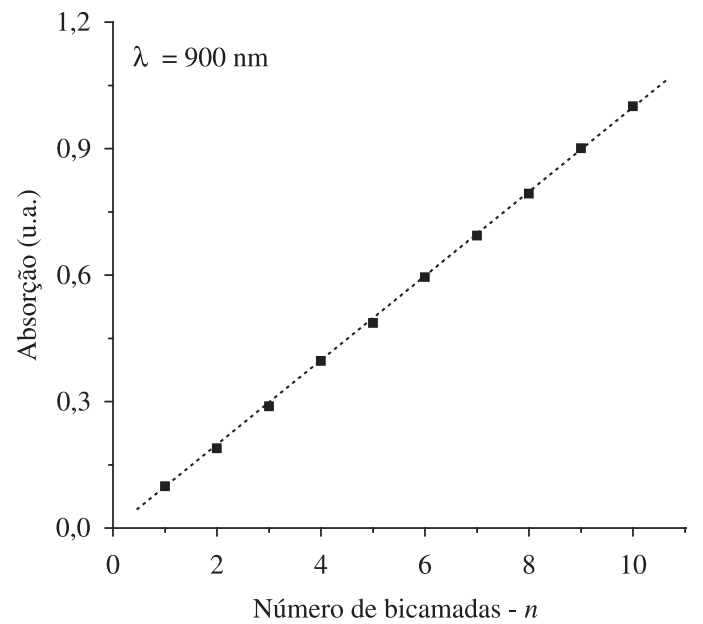

(b)

Figura 2. a) Curvas de absorção dos filmes com $n$ bicamadas e b) absorção em 900 nm, ambas em função de $n$.

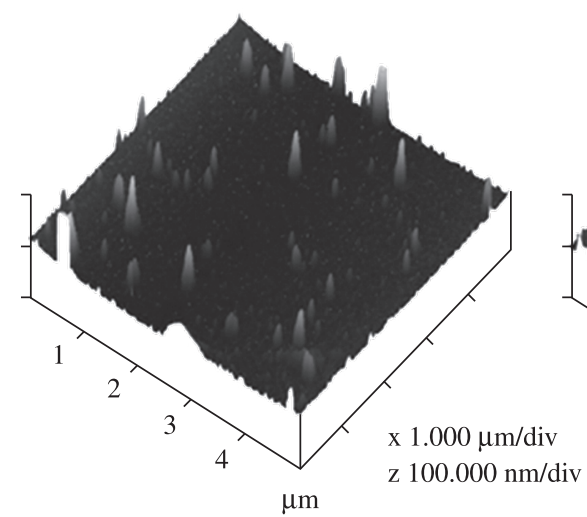

(a)

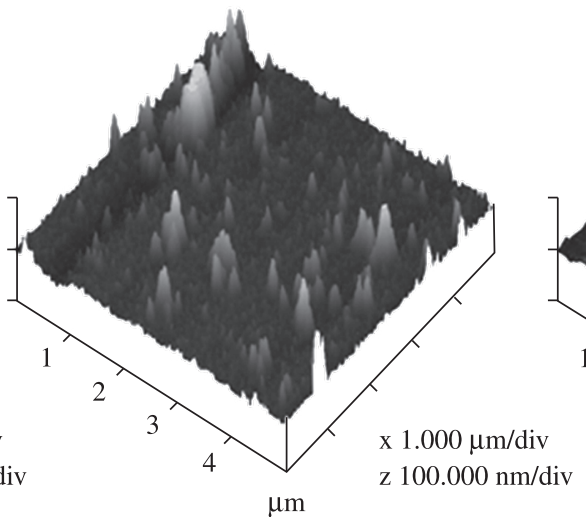

(b)

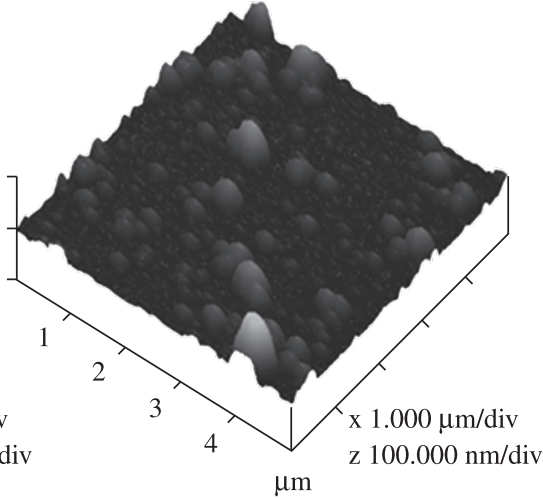

(c)

Figura 3. Imagens de AFM de filmes com a) $n=1$, b) 4 e c) 10 .

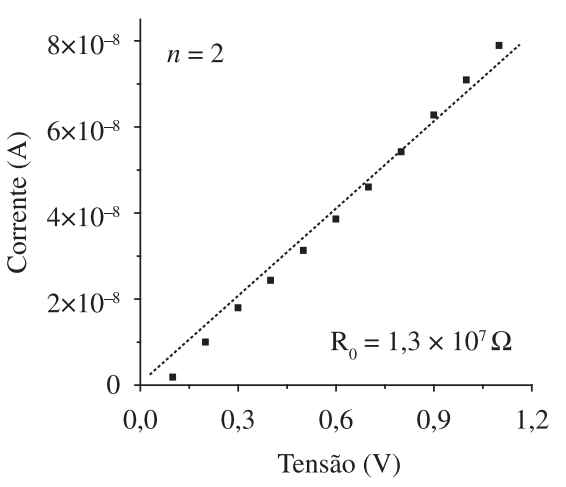

(a)

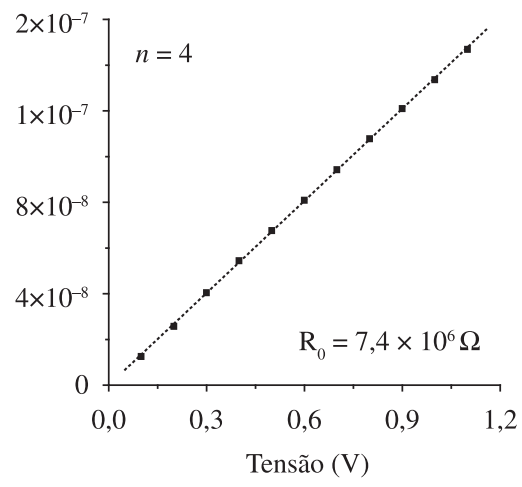

(b)

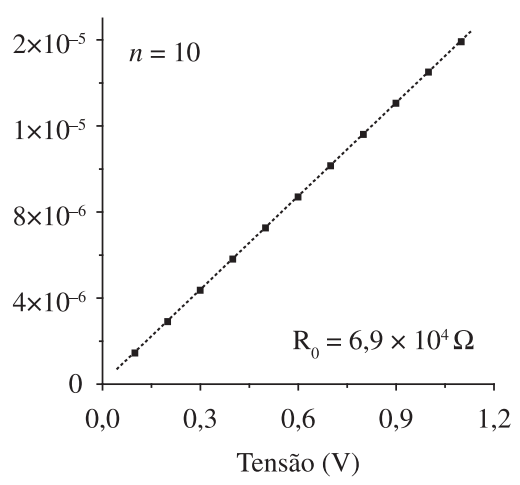

(c)

Figura 4. Curvas I vs. V, em escalar linear, obtidas de amostras com filmes de a) 2, b) 4 e c) 10 bicamadas de PANI/PVS. 


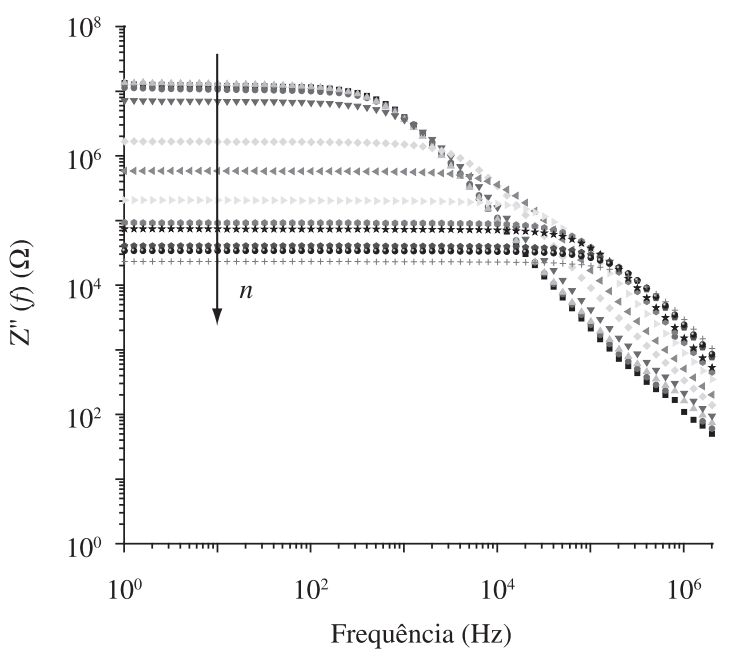

(a)

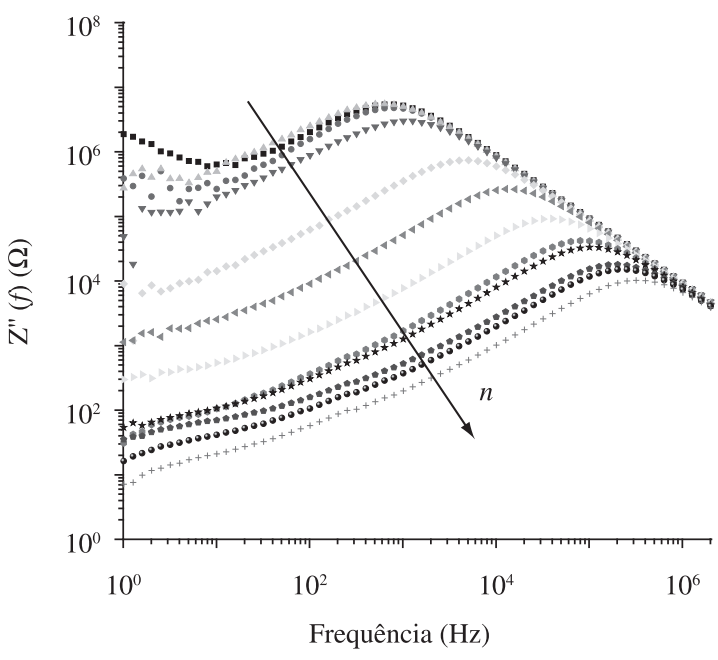

(b)

Figura 5. Gráficos experimentais das componentes a) real, $Z^{\prime}(f)$, e b) imaginária, $Z^{\prime \prime}(f)$, da impedância complexa de filmes com $n$ variando de 1 em 1 até 13.

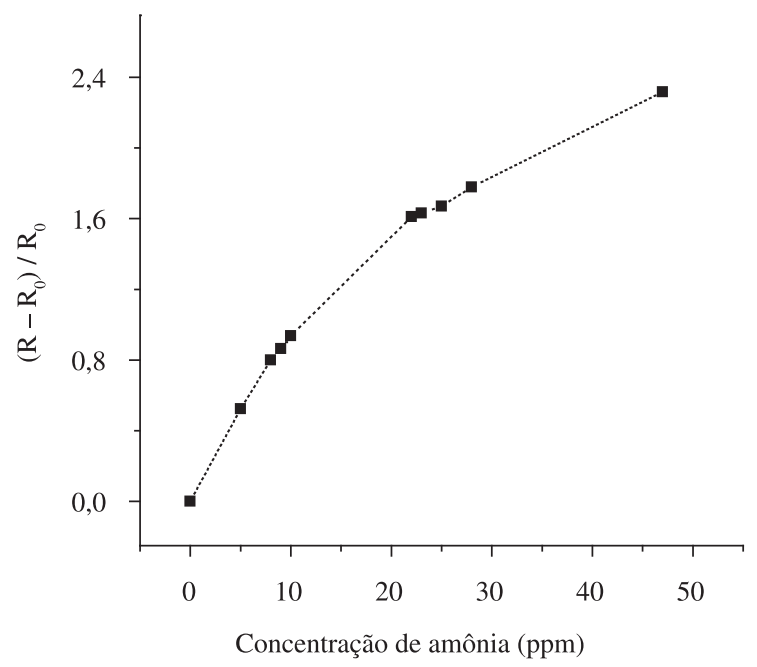

Figura 6. Resistência elétrica do dispositivo vs. variação da concentração de amônia em sistema com as mesmas condições ambientais de criação de frangos em galpões avícolas.

A Figura 4 mostra as curvas corrente $v s$. tensão $(I-V)$ obtidas de filmes com $n=2,4$ e 10 que apresentam dependência linear entre a corrente elétrica $I$ que flui pelo sistema e a tensão $V$ aplicada entre seus eletrodos, ou seja, os contatos metal/polímero comportam-se como neutros (ôhmicos) na faixa de tensão aplicada ${ }^{[14]}$ independentemente da espessura do filme. Esse comportamento linear de $I v s$. $V$ permitiu obter a resistência elétrica $\left(R_{0}\right)$ dos filmes de acordo com a Lei de $\mathrm{Ohm}$, que apresentou uma variação de aproximadamente duas ordens de grandeza quando $n$ passou de 4 para 10 .

A Figura 5 mostra as curvas de Z' $(f)$ e de Z' $(f) v s . f$ para filmes com $1 \leq n \leq 13$. Observa-se na Figura 5a que a impedância $d c$, definida como $Z_{d c}=Z^{\prime}(f \rightarrow 0)$, permanece aproximadamente constante quando $n$ varia de 1 a 4 e diminui $c a$. de 2 ordens de grandeza quando $n$ varia de 5 a 13 . É importante destacar que os valores de $Z_{d c}$ obtidos a partir de $Z^{\prime}(f \rightarrow 0)$ corroboram com os valores de $R_{0}$ encontrados a partir de medidas $d c$, Figura 4. A diminuição de $Z_{d c}$ com $n$, associada ao aumento de $f_{c}\left(=f\right.$ quando $\left.\left|Z^{\prime}\right|=\left|Z^{\prime}\right|\right)$, para filmes com $n>4$ é típica de sistemas que apresentam variações de corrente elétrica de material isolante para material condutivo ${ }^{[2]}$. Finalmente, a fraca dependência de $Z_{d c}$ com $n$, para $n \leq 4$, sugere uma significativa contribuição da interface NiCr-PANI/PVS às componentes real e imaginária da impedância complexa do sistema ${ }^{[15]}$. Essa contribuição domina as propriedades elétricas dos filmes com $n \leq 4$.

A Figura 6 mostra a variação da resistência elétrica relativa do dispositivo de filmes ultrafinos de PANI/PVS com a concentração de amônia, $\left[\mathrm{NH}_{3}\right]$, sendo $R_{0}$ o valor da resistência para $\left[\mathrm{NH}_{3}\right]=0$ e $R$ o valor da resistência para $\left[\mathrm{NH}_{3}\right] \neq 0$. Observa-se considerável sensibilidade dos filmes a baixas concentrações de amônia $(<50 \mathrm{ppm})$ tendo sua resistência elétrica relativa, $\left(\left(R-R_{0}\right) / R_{0}\right)$, aumentada cerca de 3 vezes quando submetido à variação de $\left[\mathrm{NH}_{3}\right]$ de 0 a $50 \mathrm{ppm}$ à temperatura ambiente e com umidade relativa em torno de $70 \%$. O comportamento da curva $\left(R-R_{0}\right) / R_{0} v s$. $\left[\mathrm{NH}_{3}\right]$ para pequenas concentrações de amônia $(<20 \mathrm{ppm})$ evidencia a possibilidade de aplicação desse sistema polimérico como sensor de detecção e monitoramento desse gás em galpões avícolas $^{[7-8]}$, bem como em aplicações agropecuárias onde a necessidade de monitoramento desse gás é de grande importância.

\section{Conclusões}

Os filmes ultrafinos de PANI/PVS preparados pela técnica de automontagem foram submetidos a caracterização óptica, morfológica e elétrica. De acordo com as curvas de absorção na região do UV-VIS, o crescimento dos filmes ocorreu de forma linear enquanto as informações obtidas das imagens de AFM mostraram o aumento da rugosidade, do tamanho de grão e do tamanho da partícula com o número de bicamadas. As curvas elétricas $d c$ e $a c$ evidenciaram uma significativa influência dos efeitos de interface nas propriedades elétricas 
do sistema para filmes com espessuras menores ou iguais a 4 bicamadas. Essa influência é atribuída à alta resistência elétrica interfacial entre $\mathrm{NiCr}$ e o filme polimérico quando comparada à resistência da interface Au-filme e do volume do polímero. Portanto, o comportamento elétrico dos sensores deve estar relacionado tanto à sua morfologia quanto aos efeitos de volume e de interface eletrodo-PANI/PVS. Finalmente, filmes ultrafinos de PANI/PVS depositados sobre microeletrodos interdigitados mostraram ter grande potencial para serem utilizados como sensores de amônia, em especial em galpões de criação avícola, uma vez que possuem facilidade de processamento e considerável resposta para concentrações de amônia entre 0 e 20 ppm.

\section{Agradecimentos}

Os autores agradecem ao INEO/CNPq, CNPq, CAPES e FAPEMIG pelo suporte financeiro e ao Dr. Marcelo de Assumpção Pereira da Silva (IFSC/USP) pela obtenção das imagens de Microscopia de Força Atômica.

\section{Referências Bibliográficas}

1. Chiang, C. K.; Finger, C. R.; Park, Y. W.; Heeger, A. J.; Shirakawa, H.; Louis, E. J.; Grau, S. C. \& MacDiarmid, A. G. - Phys. Rev. Let., 39, p. 1098 (1977).

2. Pope, M. \& Swenberg, C. E. - "Electronic process in organic crystals and polymers", vol. 2, Oxford University Press, Oxford, p. 1182 (1999).

3. Fishchuk, I. I.; Arkhipov, V.I.; Kadashchuk, A.; Heremans, P. \& Baessler, H. - Phys. Rev. B, 76 (2007).

4. Arkhipov, V. I.; Heremans, P.; Emelianova, E. V. \& Baessler, H. - Phys. Rev. B, 71 (2005).

5. Espinosa-González, C.; Moggio, I.; Arias-Marin, E.; Romero-Garcia, J.; Cruz-Silva, R.; Le-Moigne, J. \& Ortiz-Cisneros, J. - Synth. Met., 139, p. 155-161 (2003).
6. Wiziack, N. K. L.; Paterno, L. G.; Fonseca, F. J. \& Mattoso, L. H. C. - Sensor Actuator B. Chem., 122, p. 484-492 (2007).

7. Dirani, E. A. T.; Regaço, M. P.; Bianchi, R. F.; Heleno, C. M. N.; Zucolotto, V.; Andrade, A. M. \& Fonseca, F. J. - "Organic material as gas sensors for farm application", in: Anais do III Encontro da SBPMat, Foz do Iguaçu, oct (2004).

8. Dirani, E. A. T.; Bianchi, R. F.; Heleno, C. M. N.; Regaço, M. P.; Zucolotto, V.; Andrade, A. M. \& Fonseca, F. J. - "Gas sensors based on organic materials", in: Proceedings of 8 European Conference on Molecular Electronics, Bologna, june (2005).

9. Owada, A. N.; Nääs, I. A.; Moura, D. J. \& Baracho, M. S. - Eng. Agríc., 27, p. 611-618. (2007).

10. Zucolotto, V.; Ferreira, M.; Cordeiro, M. R.; Constantino, C. J. L.; Moreira, W. C. \& Oliveira Jr., O. N. - Sensor Actuator B. Chem., 113, p. 809 (2006).

11. Oliveira, M. C.; Almeida, C. V.; Andrade, D. O. \& Rodrigues, S. M. M. - Rev. Bras. Zootec., 32, p. 951-954 (2003).

12. Ram, M. K.; Salermo, M.; Adami, M.; Faraci, P. \& Nicolini, C. - Langmuir, 15, p. $1252-1259$ (1999).

13. Lobo, R. F. M.; Silva, M. A. P.; Raposo, M. \& Faria, R. M. - Nanotechnology, 14, p. 101-108 (2003).

14. Szymanski, A. \& Bak, G. W. - J. Phys. D Appl. Phys., 19, p. 25-28 (1986).

15. Santos, M. C. - "Preparação e caracterização elétrica de filmes ultrafinos de polianilina e poli (vinil sulfato de sódio) (PANI/PVS)" Master Thesis, Universidade Federal de Ouro Preto, Ouro Preto (2009).

Enviado: 09/10/09

Reenviado: 22/12/09

Aceito: $07 / 01 / 10$

DOI: $10.1590 / \mathrm{S} 0104-14282010005000018$ 